\title{
3D Superlithiophilic Interphase for Dendrite-Free Lithium Metal Anodes
}

Yun Qiao,, a d Qingling Li, a, d Xin-Bing Cheng, ${ }^{\text {b }}$ Fuxia Liu, ${ }^{c}$ Yange Yang, ${ }^{a, d}$

Zhansheng Lu, ${ }^{c}$ Jin Zhao, a, d Jiawei Wu, a, d He Liu, ${ }^{b}$ Shuting Yang, ${ }^{\text {a, d }}$ Yang $\mathrm{Liu}^{* a}$

a School of Chemistry and Chemical Engineering, Henan Normal University, Xinxiang 453007, China

b Beijing Key Laboratory of Green Chemical Reaction Engineering and Technology, Department of Chemical Engineering, Tsinghua University, Beijing 100084, China

${ }^{c}$ College of Physics and Materials Science, Henan Normal University, Xinxiang 453007, China

d National and Local Joint Engineering Laboratory of Motive Power and Key Materials, Xinxiang 453007, China

E-mail: cxb12@mails.tsinghua.edu.cn (Xin-Bing Cheng); shutingyang@,foxmail.com (Shuting Yang); liuy986@163.com (Yang Liu) 
Table S1. EDS elemental analysis of NCNF and NCNF@ZnO films.

\begin{tabular}{ccccc}
\hline Samples & $\mathrm{C}(\mathrm{wt} \%)$ & $\mathrm{N}(\mathrm{wt} \%)$ & $\mathrm{O}(\mathrm{wt} \%)$ & $\mathrm{Zn}(\mathrm{wt} \%)$ \\
\hline NCNF & 76.06 & 14.54 & 9.40 & 0 \\
NCNF@ZnO & 44.72 & 16.31 & 14.49 & 24.48 \\
\hline
\end{tabular}




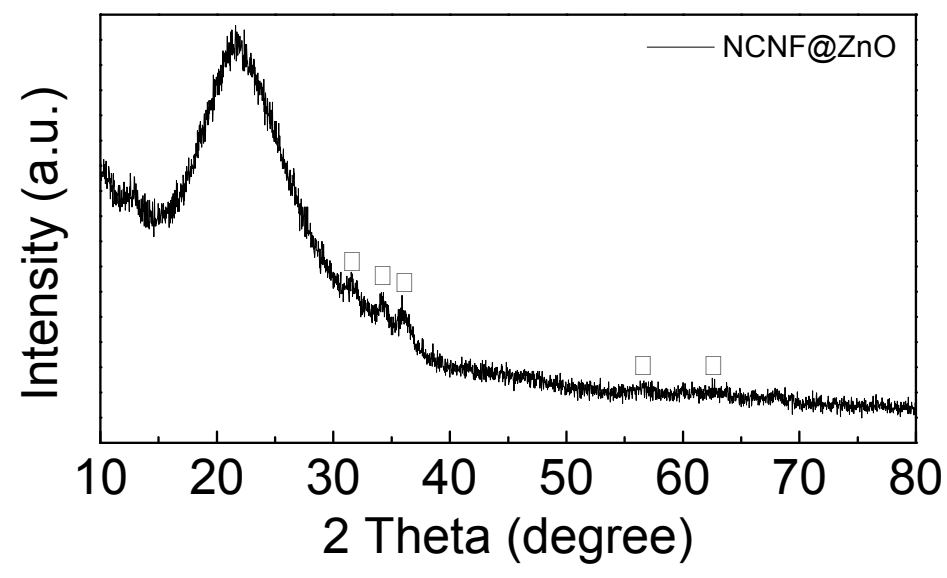

Figure S1. XRD patterns of the NCNF@ZnO sample 

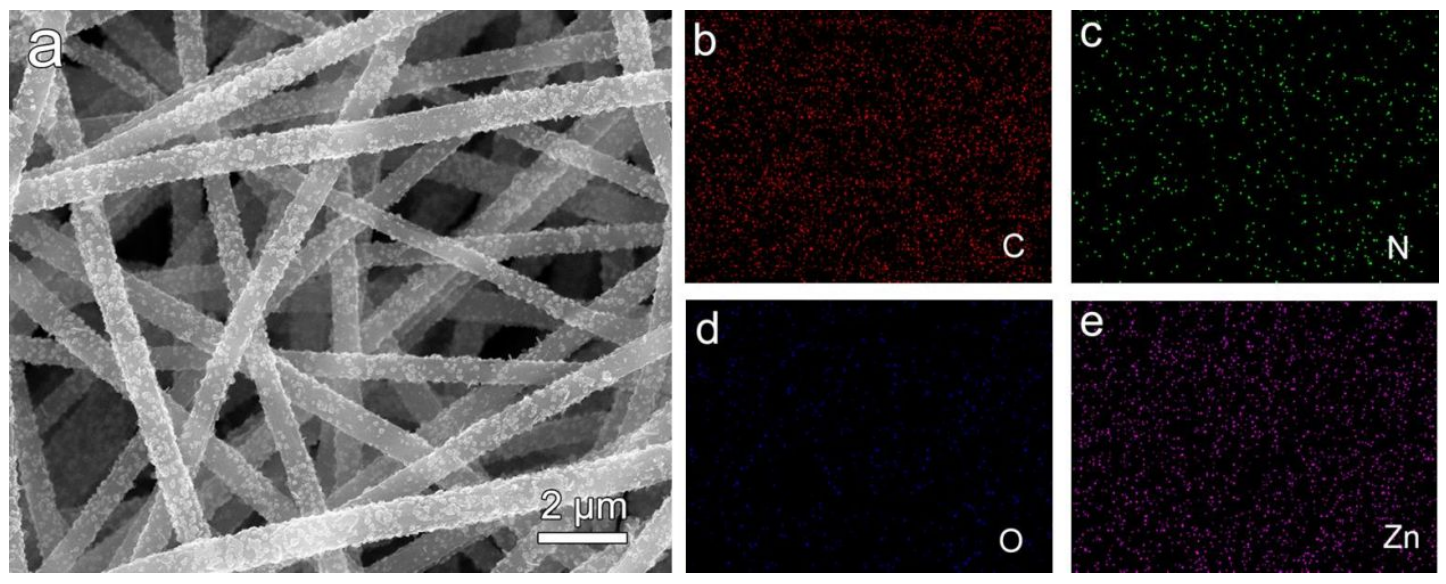

Figure S2. SEM image of (a) NCNF@ZnO and the corresponding EDS elemental maps of (b) carbon, (c) nitrogen, (d) oxygen and (e) zinc. 

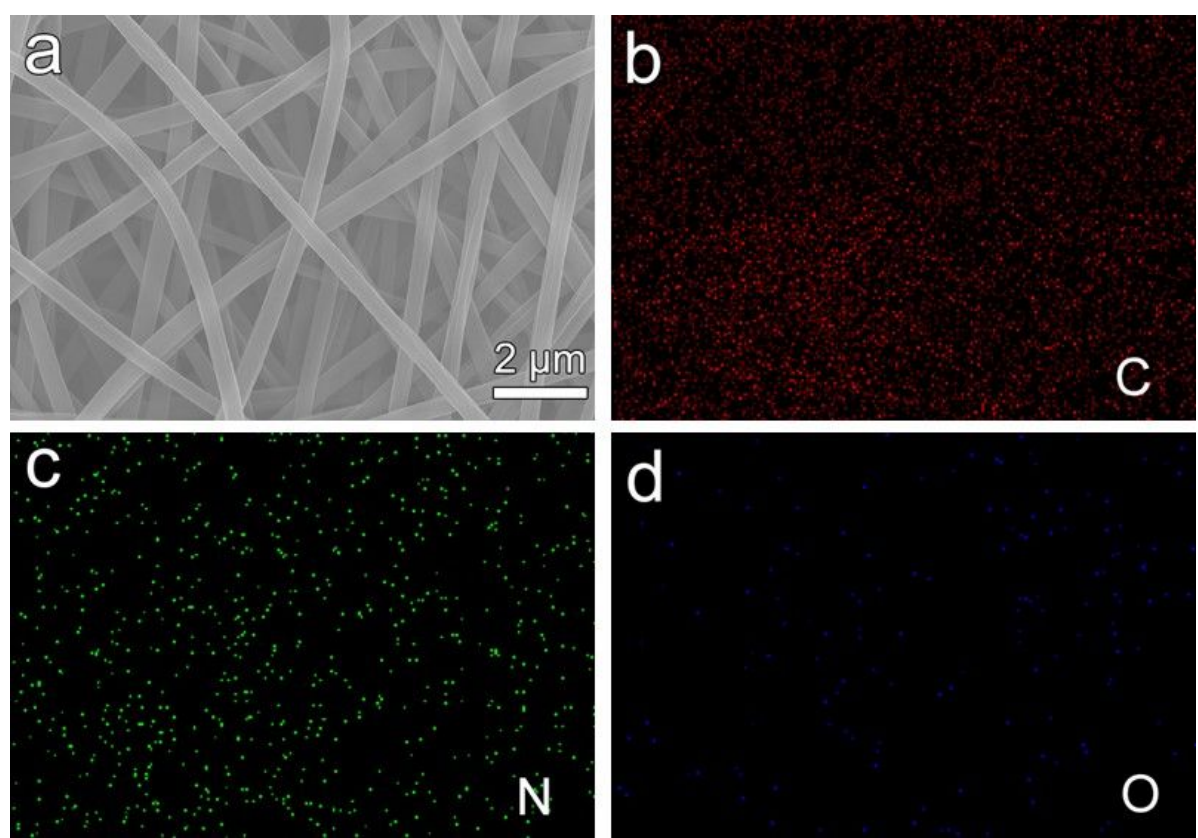

Figure S3. SEM image of (a) NCNF and the corresponding elemental maps of (b) carbon, (c) nitrogen and (d) oxygen. 


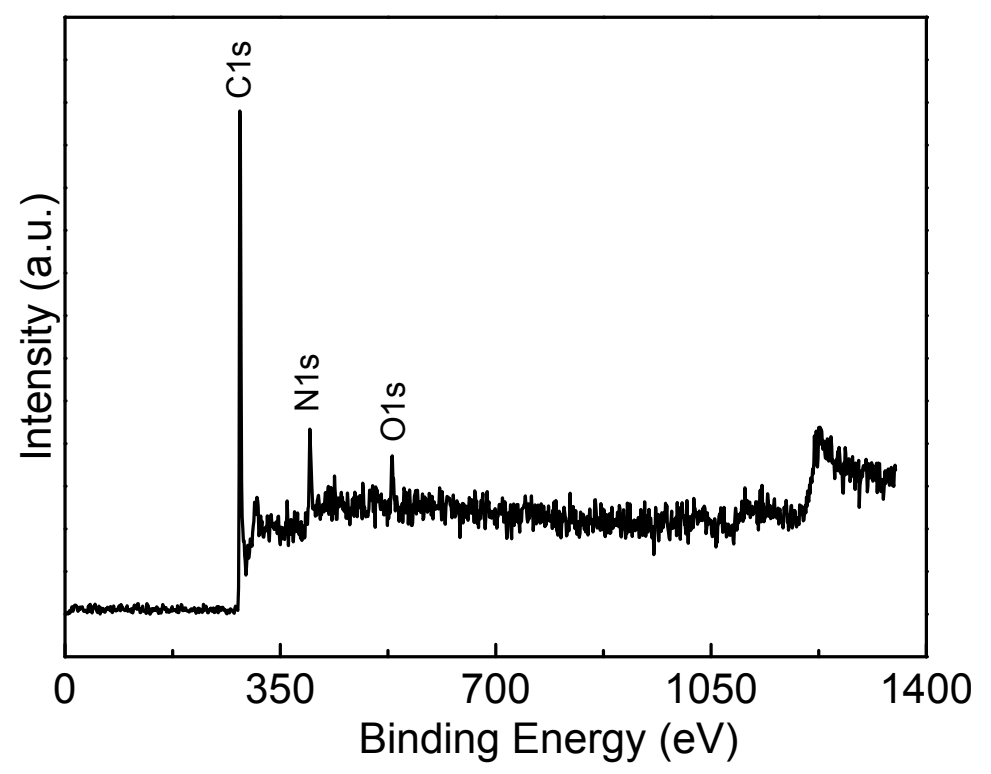

Figure S4. The survey XPS spectrum of NCNF. 

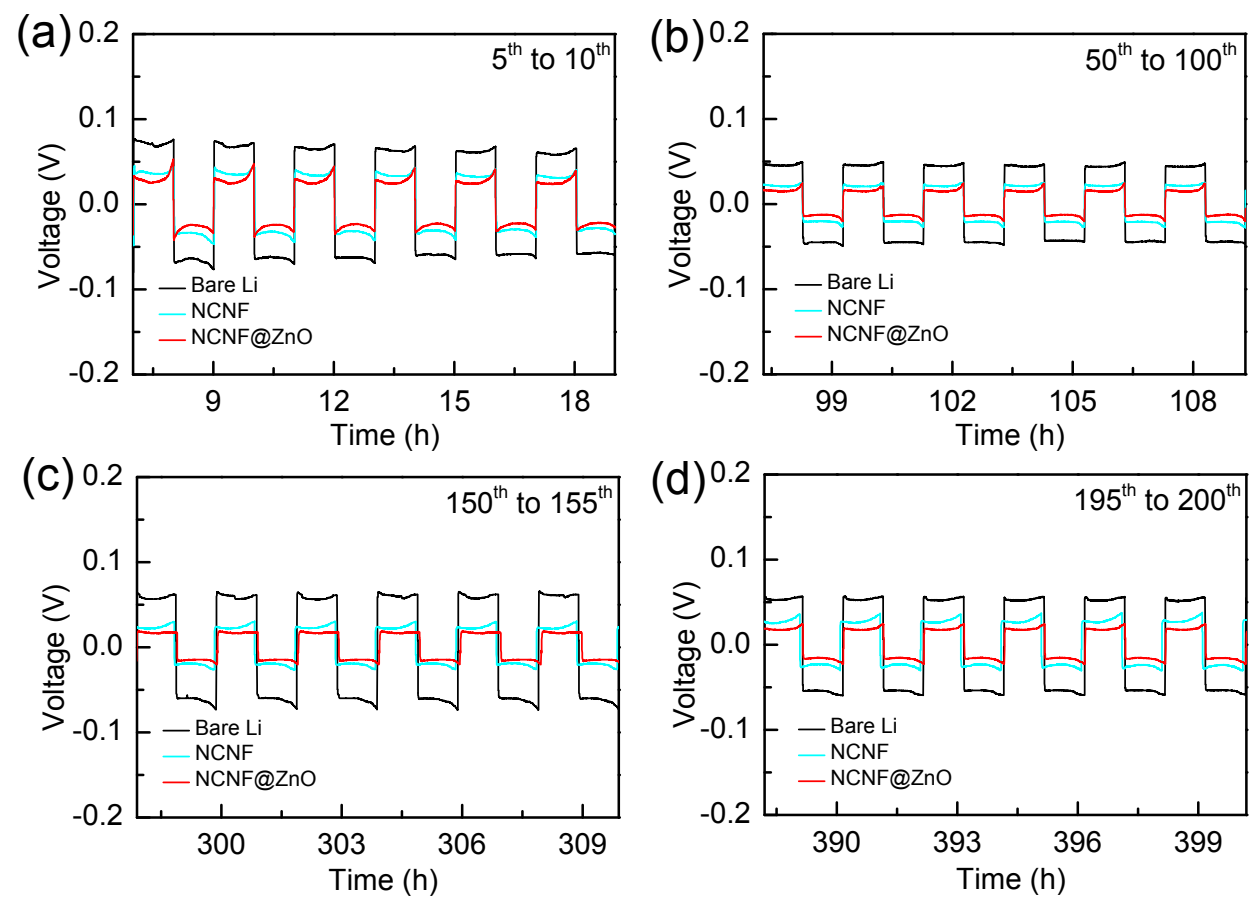

Figure S5. The detailed voltage-time profiles at different cycles for bare $\mathrm{Li} / \mathrm{Li}$ symmetric cell, Li/Li cell with NCNF interlayer and Li/Li cell with 3D CNF@ZnO as the lithiophilic interlayer at a current density of $1 \mathrm{~mA} \mathrm{~cm}-2$ with a stripping/plating capacity of $1 \mathrm{~mA} \mathrm{~h} \mathrm{~cm}^{-2}$, (a) $5^{\text {th }}-10^{\text {th }}$, (b) $50^{\text {th }}-100^{\text {th }}$, (c) $150^{\text {th }}-155^{\text {th }}$, (d) $195^{\text {th }}-200^{\text {th }}$. 

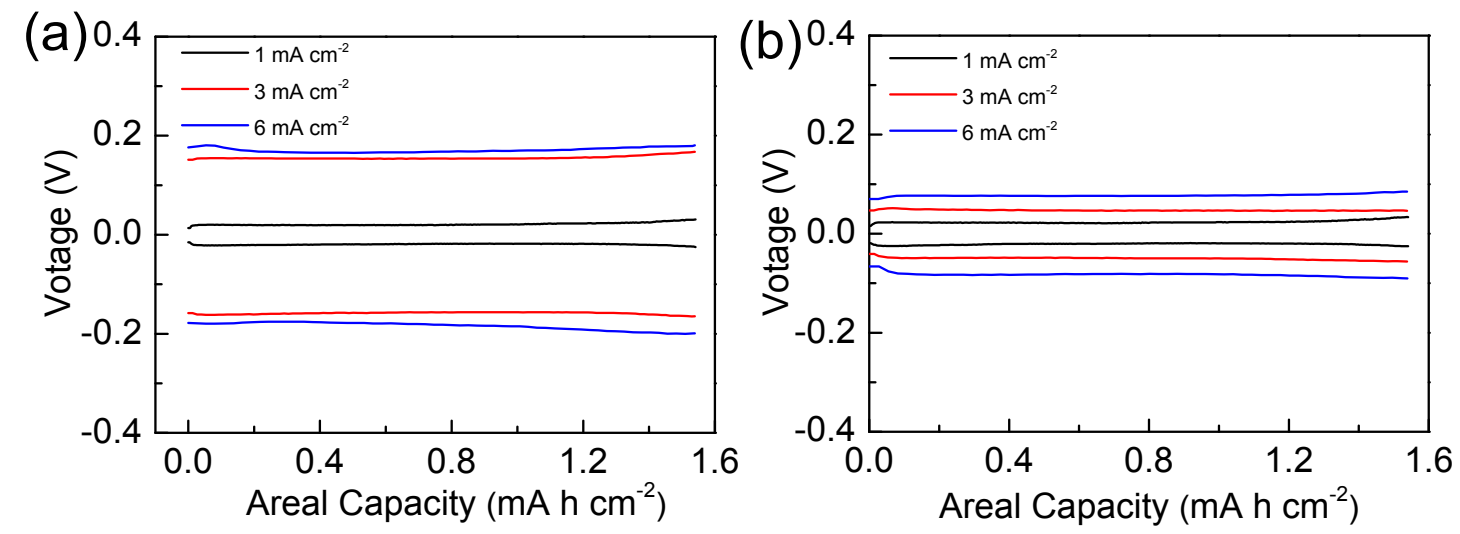

Figure S6. Voltage profiles of (a) bare Li metal cells and (b) cells with NCNF at different current densities; ebviously, cells with NCNF@ZnO show stable voltage profiles with smaller hysteresis. 

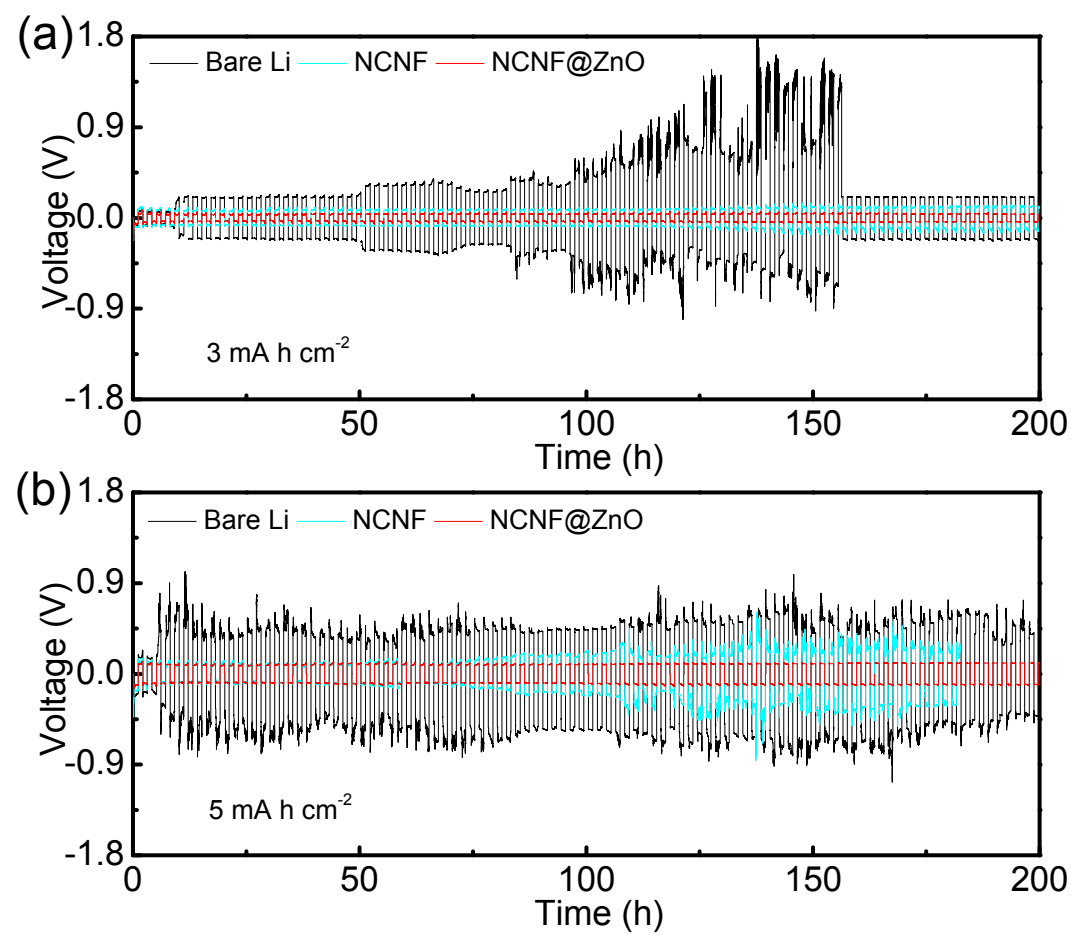

Figure S7. Galvanostatic cycling performance of symmetric cells using bare Li-metal electrode (black), with NCNF@ZnO interlayer (red) and with pristine NCNF interlayer (cyan) with a deposition/dissolution capacity of $3 \mathrm{~mA} \mathrm{~h} \mathrm{~cm}^{-2}$ (a) and $5 \mathrm{~mA} \mathrm{~h} \mathrm{~cm}{ }^{-2}$ (b).

The cycling performance of symmetric cells were performed at higher capacities of 3

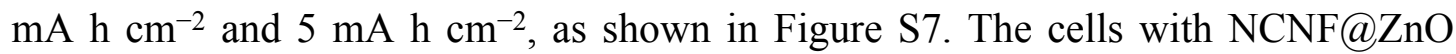
interlayer deliver a low and stable overpotentials of $\sim 42 \mathrm{mV}$ and $\sim 101 \mathrm{mV}$ with a capacity of 3 mA h cm${ }^{-2}$ after $150 \mathrm{~h}$, respectively. In contrast, the overpotential of the cell with NCNF interlayer is as high as $105 \mathrm{mV}$ after depositing Li for $3 \mathrm{~mA} \mathrm{~h} \mathrm{~cm}{ }^{-2}$. Meanwhile, the bare Li symmetrical cells suffer severe voltage fluctuation, no matter the deposition/dissolution capacities are of 3 or $5 \mathrm{~mA} \mathrm{~h} \mathrm{~cm}^{-2}$. 
(a)

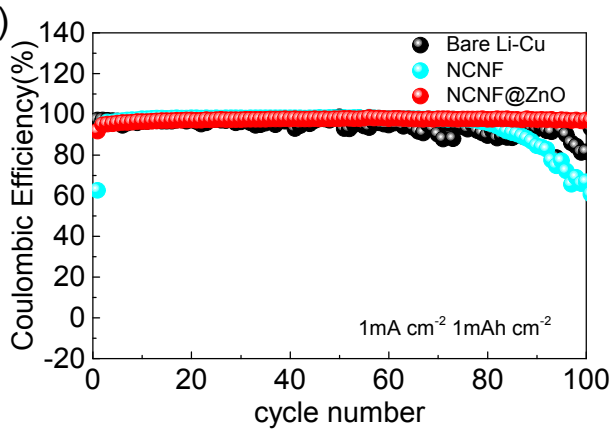

(b)

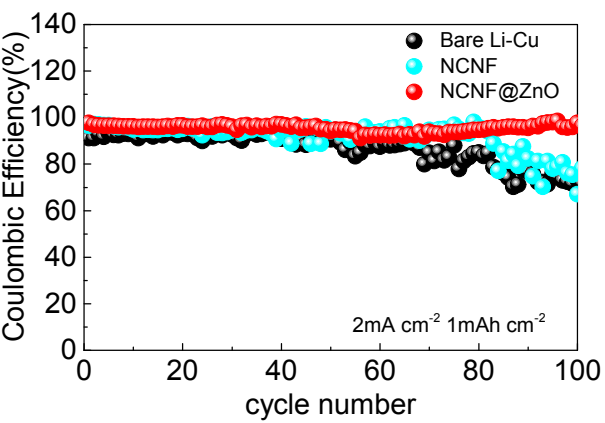

Figure S8. Coulombic efficiency obtained from $\mathrm{Li} / \mathrm{Cu}$ cells at a current density of (a) $1 \mathrm{~mA} \mathrm{~cm}^{-2}$, (b) $2 \mathrm{~mA} \mathrm{~cm}^{-2}$ with a total capacity of $1 \mathrm{~mA} \mathrm{~h} \mathrm{~cm}$. $^{-2}$. 

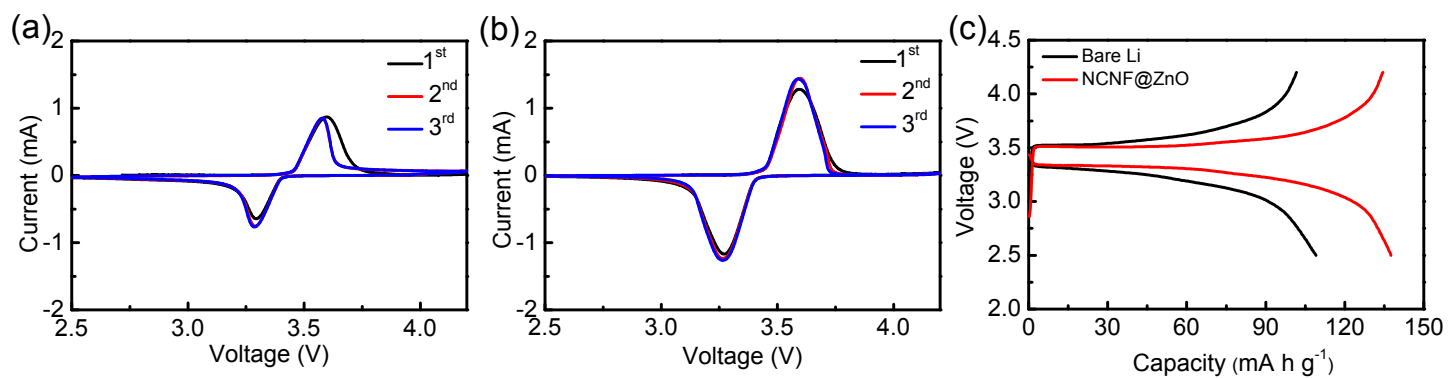

Figure S9. Cyclic voltammetry (CV) curves of the Li|LFP cells (a) without interlayer, (b) with NCNF@ZnO interlayer in the voltage window of 2.5-4.2 V at a scan rate of $0.1 \mathrm{mV} \mathrm{s}-1$. (c) The charge-discharge profiles of the Li|LFP full cells with/without interlayer at $1 \mathrm{C}$. 

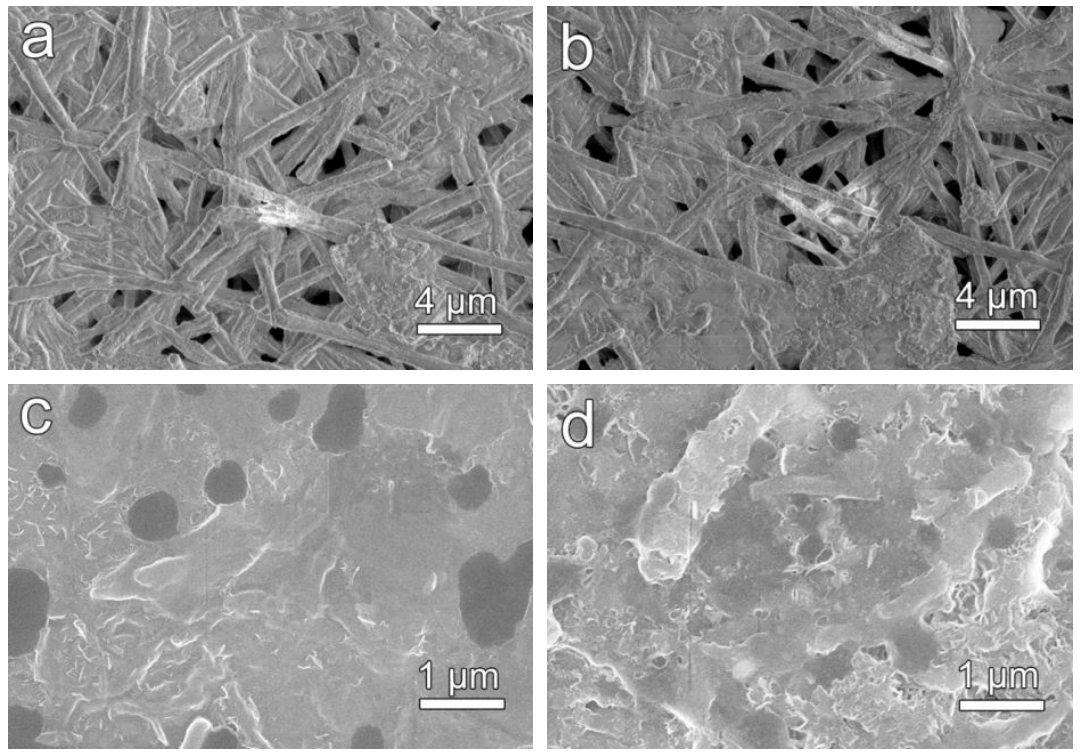

Figure S10. Top-view SEM images of the NCNF after 15 cycles at a current density of (a) $3 \mathrm{~mA} \mathrm{~cm}{ }^{-2}$, (b) $6 \mathrm{~mA} \mathrm{~cm}^{-2}$, respectively. Top-view SEM images of the Li metal with the NCNF interlayer after 15 cycles at a current density of (c) $3 \mathrm{~mA} \mathrm{~cm}{ }^{-2}$, (d) 6 $\mathrm{mA} \mathrm{cm}{ }^{-2}$, respectively. 

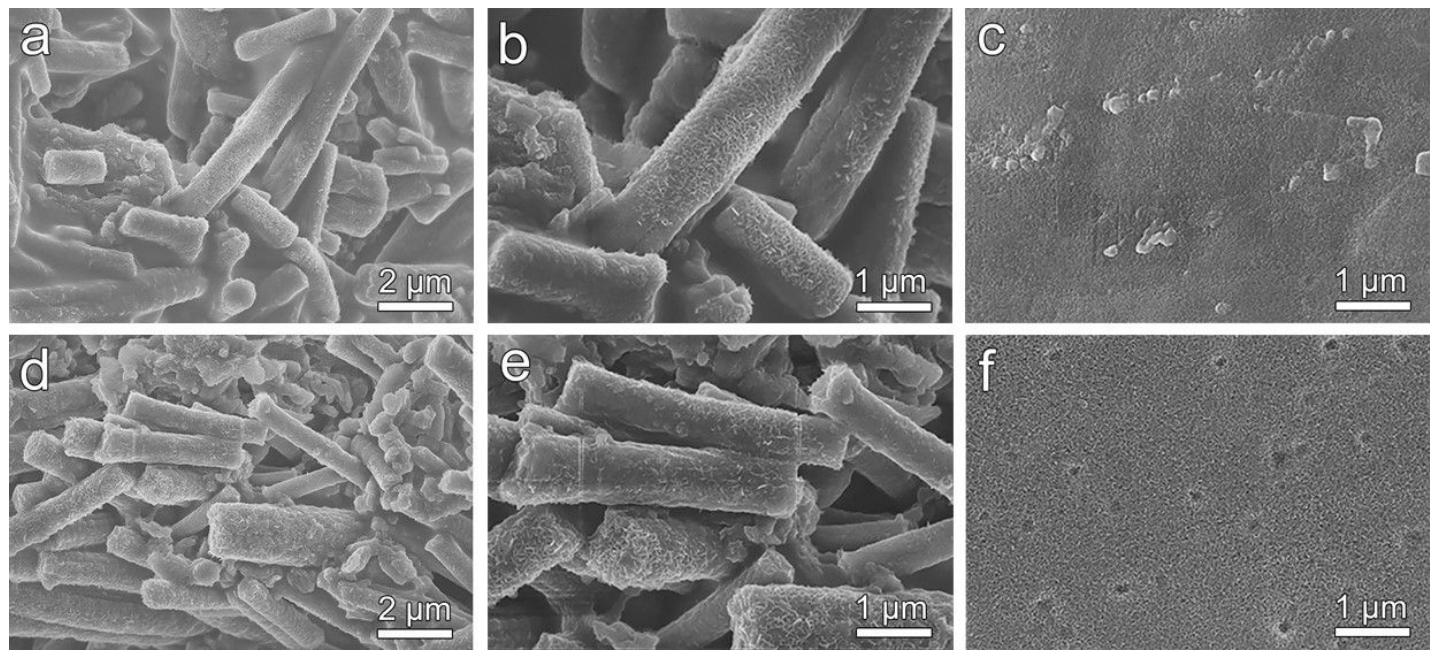

Figure S11. SEM images of (a, b) the NCNF@ZnO interlayer and (c) Li metal anode after 15 cycles at a current density of $3 \mathrm{~mA} \mathrm{~cm}^{-2}$ in the cell with NCNF@ZnO interlayer. SEM images of (d, e) the NCNF@ZnO interlayer and (f) Li metal anode after 15 cycles at a current density of $6 \mathrm{~mA} \mathrm{~cm}-2$ in the cell with NCNF@ZnO interlayer. 

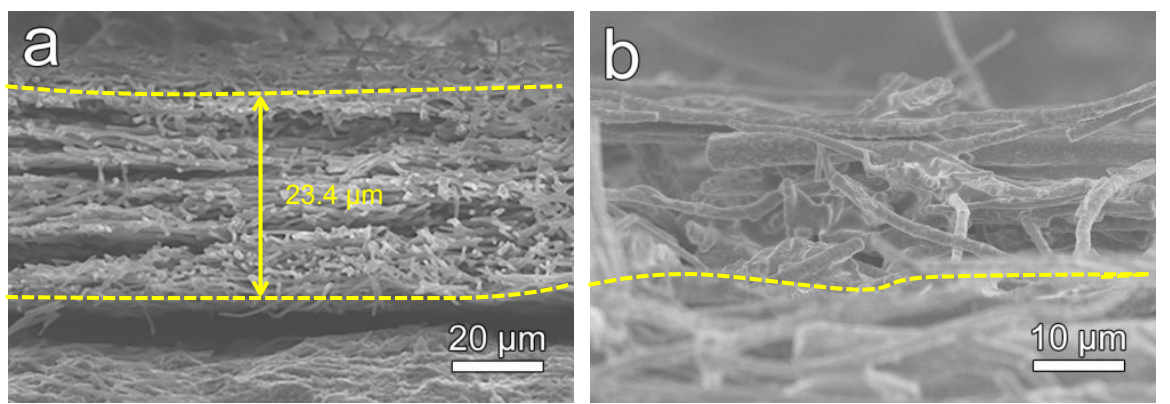

Figure S12. Cross-sectional SEM images of $(a, b)$ NCNF@ZnO interlayer after 15 cycles at a current density of $1 \mathrm{~mA} \mathrm{~cm}^{-2}$, with a capacity of $1 \mathrm{~mA} \mathrm{~h} \mathrm{~cm}{ }^{-2}$. 